\title{
On the Numerical Accuracy of the Boundary Element Method
}

\author{
JAN W. H. MEIJS, ONNO W. WEIER, MARIA J. PETERS, AND ADRIAAN VAN OOSTEROM
}

\begin{abstract}
The numerical accuracy of the boundary element (BE) method used to solve the volume conduction problem of nested compartments, each having a homogeneous conductivity, is studied. The following techniques for improving this accuracy are discussed: the handling of the auto solid angle element $\Omega^{i i}$, the overall refinement of the level of discreteness, the use of a locally refined discrete grid, the isolated problem approach, and an adaptive refined computation of the discrete surface integrals involved in the BE method. The effects of these techniques on the numerical accuracy of the computed electrical potentials are illustrated by taking a volume conductor consisting of four concentric spheres representing the head since for this model an analytical (exact) solution is available. The techniques are of importance for numerically computed electroencephalograms (EEG's) since the numerically computed surface EEG's are severely affected by the relatively low conductivity of the compartment representing the skull.
\end{abstract}

\section{INTRODUCTION}

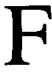
R a quantitative interpretation of electroencephalograms (EEG's) and magnetoencephalograms (MEG's) in terms of the underlying brain activity, mathematical models are used to describe both the source of this activity and the head as a volume conductor. For this purpose, a single current dipole and a four concentric spheres model are commonly used since for these models analytical expressions are available for computing both the EEG and the MEG [1]. By means of inverse techniques, equivalent sources can be estimated from measured EEG's or MEG's. A more realistically shaped model of the head [2]-[4] might result in better fits between measured and estimated data since it describes the anatomical reality better (Fig. 1).

However, during simulation studies using a head model consisting of four realistically shaped compartments, the discrete boundary element (BE) method used to compute the EEG's was found to generate numerical errors, which were blown up due to the small conductivity of the skull [5]. To study this problem, simulations of EEG's based on the four concentric spheres model were carried out by means of the BE method. Comparison of the results with those obtained by an analytic method [6] showed large discrepancies. The differences in the field patterns due to

Manuscript received April 15, 1988; revised July 19, 1988

J. W. H. Meijs and O. W. Weier are with the Department of Electrical Engineering, Twente University, 7500 AE Enschede. The Netherlands.

M. J. Peters is at the Department of Physics, Twente University, 7500 AE Enschede, The Netherlands.

A. van Oosterom is with the University of Nijmegen, $6700 \mathrm{HB}$ Nijmegen, The Netherlands.

IEEE Log Number 8929824

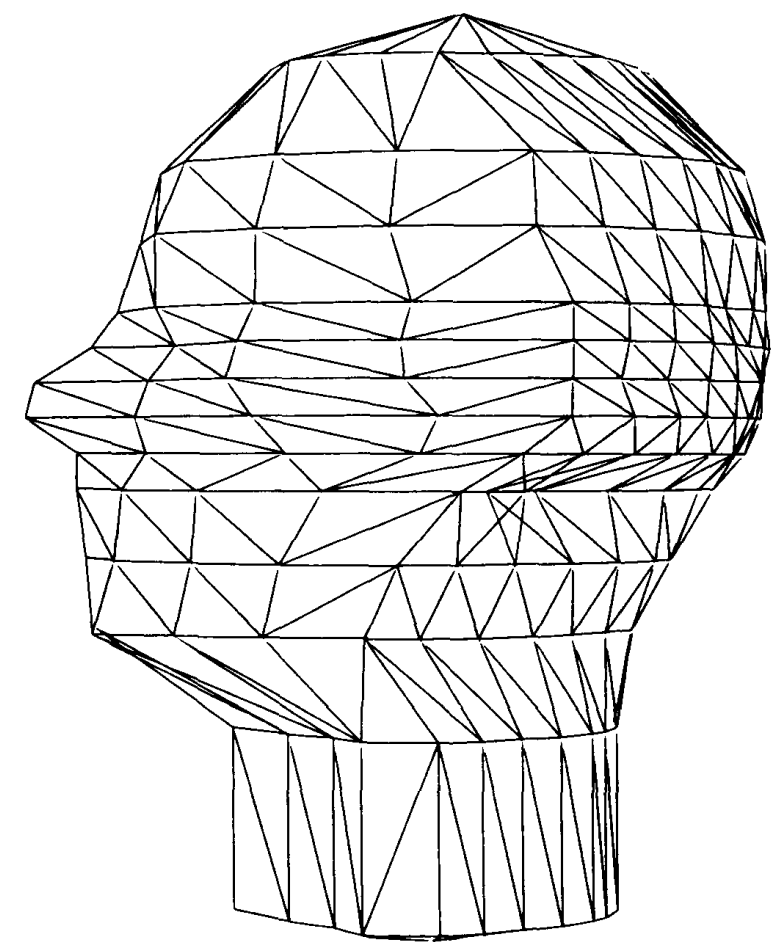

Fig. 1. Triangularly paneled model of the outer compartment of the realistically shaped head as constructed by Meijs et al. [2].

the use of the realistically shaped head model rather than the four concentric spheres model are in the same order of magnitude [7]. As a consequence, no quantitative analysis could be made of the influence of realistically shaped head models on the EEG.

Although the numerical errors can be reduced by increasing the number of discretization points, this increase will result in extensive computation time and storage demands. To restrict the numerical effort and nevertheless increase the numerical accuracy, the following techniques have been studied in this paper: the approximation of the auto solid angle element $\left(\Omega^{i i}\right)$ is improved, a locally refined triangular grid is used and the magnification of the numerical errors is eliminated by means of the isolated problem (IP) approach, and finally, an adaptive refined computation of the discrete surface integrals is studied. Although Hämäläinen and Sarvas [8] previously found that for their three spheres model the numerical errors in 
the EEG's could be reduced to an acceptable level by just using the IP approach, it will be shown that the numerical errors using a four spheres model are still relatively large and need to be further reduced by the techniques discussed in this paper.

The numerical techniques described in this paper will be studied for a triangulated four concentric spheres model of the head since for this model an analytical expression is available for the computation of the EEG's [6] and, hence, the numerical errors in the numerically computed EEG's can be quantified.

\section{THEORY}

\section{A. The Boundary Element Method}

The potential distribution at each boundary surface $S_{k}$ within an arbitrary shaped multicompartment model, in which each compartment has a homogeneous and isotropic conductivity, can be computed [9] as

$$
V_{k}\left(\boldsymbol{r}^{\prime}\right)=\frac{\sigma_{s}}{\bar{\sigma}_{k}} V_{k}^{\infty}\left(\boldsymbol{r}^{\prime}\right)-\frac{1}{4 \pi \bar{\sigma}_{k}} \sum_{l=1}^{N} \Delta \sigma_{l} \int_{S_{l}} V_{l}(\boldsymbol{r}) d \Omega_{k l}
$$

with

$$
d \Omega_{k l}=\frac{\left(\boldsymbol{r}^{\prime}-\boldsymbol{r}\right)}{\left|\boldsymbol{r}^{\prime}-\boldsymbol{r}\right|^{3}} \cdot \boldsymbol{n} d S
$$

where

$$
\begin{aligned}
\sigma_{s}= & \text { the conductivity in the source region, } \\
V_{k}^{\infty}\left(\boldsymbol{r}^{\prime}\right)= & \text { the electrical potential which arises from } \\
& \text { the source, located in an infinite homoge- } \\
& \text { neous medium with conductivity } \sigma_{s}, \\
d \Omega_{k l}= & \text { the solid angle subtended by the surface } \\
& \text { element } \boldsymbol{n} d S \text { in } r \text { on surface } S_{l} \text { at a point } \\
& \text { of observation } \boldsymbol{r}^{\prime} \text {, located on } S_{k}, \\
\sigma_{k}^{-}= & \text {the conductivity just inside surface } S_{k}, \\
\sigma_{k}^{+}= & \text {the conductivity just outside surface } S_{k}, \\
\Delta \sigma_{k}= & \sigma_{k}^{-}-\sigma_{k}^{+}, \\
\bar{\sigma}_{k}= & \frac{1}{2}\left(\sigma_{k}^{-}+\sigma_{k}^{+}\right), \\
N= & \text { the total number of compartment bound- } \\
& \text { aries. }
\end{aligned}
$$

Equation (1) shows that the contributions of the volume currents to the potential distribution in a piecewise homogeneous volume conductor can be considered to be equivalent to those resulting from current dipole layers (double layers) at the compartment boundaries in a medium with conductivity $\bar{\sigma}_{k}$. These dipole layers are also called the "secondary sources." The orientation of these secondary dipolar sources is normal to the interfaces and their strengths are proportional to the local potential and to the conductivity differences between the successive compartments. The electrical potential due to these secondary sources is added to the electrical potential caused by the primary source, thus setting up the total observed potential. The Fredholm integral equation of the second kind, denoted in (1), must be solved in order to find the potential distributions $V_{k}$ on the surfaces $S_{k}$. A numerical solution can be obtained by decomposing the closed sur- faces $S_{l}$ into $N_{l}$ triangular panels $\Delta_{l}^{j}\left(j=1 \cdots N_{l}\right)$. The potential on each triangle is considered to be a constant for which the potential at the center of mass of the triangles is taken, called the "center of mass" approach [10] The solid angle $\Omega_{k l}^{i j}$ (1) subtended by a triangle $\Delta_{l}^{j}$ with respect to the center of mass of triangle $\Delta_{k}^{i}$ can be computed exactly if the locations of the vertices are known [11].

The boundary element method handles the discrete version of integral (1) can be rewritten as the linear expression:

$$
v=g+B v .
$$

The elements of matrix $\boldsymbol{B}$ and of the vectors $\boldsymbol{v}$ and $g$ are

$v^{i}=$ the potential value in the center of mass of the $i$ th triangle $\left(\Delta_{i}\right)$,

$g^{i}=$ the potential due to the source in the center of mass of $\Delta_{i}$,

$B^{i j}=$ the matrix element which is proportional to the solid angle subtended by $\Delta_{j}$ at the center of mass of $\Delta_{i}$.

Matrix $\boldsymbol{B}$ has an eigenvector with eigenvalue one ( $\lambda=$ 1). After eliminating this singularity as well as the other known eigenvectors usually having a high eigenvalue $(|\lambda|$ $\approx 1$ ) from this matrix $\boldsymbol{B}$, by means of a multideflation technique [12], the linear system (2) can be solved iteratively by using the Gauss-Seidel or the Jacobi iteration scheme [13].

If the volume conductor involves more than one nested compartment, numerical errors will become apparent. These errors are due to the limited validity of the two assumptions inherent to the BE method: 1) the potential is assumed to be constant over each triangle and 2) the auto solid angle element $\left(\Omega^{i i}\right)$, which is the solid angle subtended by a triangle at a point of that same triangle, is $-2 \pi$.

In the next sections these discretization problems will be discussed.

\section{B. The Improved $\Omega^{i i}$ Approximation}

In the center of mass approach of the BE method, as described above (2), the surface boundaries are discretized using triangular panels and the centers of mass of these triangles are referred to as the discretization points. The potential over each triangle $\Delta_{j}$ is assumed to be constant and the discretized surface integral of (1) is approximated by

$$
\int_{\Delta_{j}} V_{l}(\boldsymbol{r}) d \Omega_{k l} \approx \Omega^{i j} v^{j}
$$

where

$\Omega^{i j}$ is the solid angle subtended by triangle $j$ at the center of mass of triangle $i$, located on surface $S_{k}(i$ $\neq j$ ). The auto solid angle element $\Omega^{i i}$ is $-2 \pi$ [12], and

$v^{j}$ is the potential value at the center of mass of triangle $\Delta_{j}$, on $S_{l}$. 
Instead of this "center of mass" approach of the BE method, we use the vertex approach in which the vertex points of the triangles rather than the centers of mass are taken as the discretization points, $v^{j}$ is the potential calculated at vertex point $j$. Barr et al. [10] already demonstrated the advantages of the latter approach. The solid angle $\Omega^{i j}$ in the vertex approach is, for $i \neq j$, defined as one third of the solid angles subtended by the triangles surrounding the vertex point $j$ at vertex point $i$ [14]. In the center of mass approach a value of $-2 \pi$ is attached to the auto solid angle element $\Omega^{i i}$ and after moving the term $-\Omega^{i i} v^{i}$ to the left-hand side of (2), the remaining diagonal elements $B^{i i}$ ( $\cong$ the remaining $\Omega^{i i}$ elements), are equal to zero. In the vertex approach, the remaining auto solid angle elements $\Omega^{i i}$ may deviate substantially from zero and can even become of the order of one, denoted by 0 ( 1 ). Therefore, a different approximation of the remaining auto solid angle $\Omega^{i i}$ will be used. This approximation has been introduced into the application of the BE technique in basic electrocardiography by one of the authors [14]. Since it has not been documented before, it will be described in Appendix VII-B, see also Fig. 2. In this approach

$$
\begin{aligned}
& \Omega^{i i}=2 \pi \frac{(\cos (\alpha)-1)}{\alpha}, \\
& \text { with } \quad \alpha=\arccos (r / R) .
\end{aligned}
$$

The total solid angle, subtended by a closed surface at a vertex point $i$ on this surface is equal to $-2 \pi$ if the associated limit is taken for the observation point approaching vertex point $i$ from the inside of the surface. A possible difference in the computation of this solid angle is distributed evenly over the vertex points surrounding the vertex point $i$.

If the vertex approach of the BE method is used, the elements of matrix $\boldsymbol{B}$ and vectors $\boldsymbol{v}$ and $\boldsymbol{g}$ of the linear system represented in (2) are

$v^{i}=$ the potential in the $i$ th vertex point,

$g^{i}=$ the source term in the $i$ th vertex point and

$B^{i j}=$ the multideflated matrix element representing the influence of the $j$ th on the $i$ th vertex point, $B^{i i} \cong$ the remaining $\Omega^{i i} \neq 0$.

\section{The Isolated Problem Approach}

If one of the components inside the volume conductor has a relatively low conductivity compared with the conductivities of the other components, the numerical errors have been shown to be blown up [5], [8]. Let us assume that only one compartment inside the volume conductor has a relatively low conductivity, not being the outer shell, i.e., the $(m-1)$ th compartment of the volume conductor consisting of $N(N>2)$, nested, compartments $(N \geq m$, Fig. 3),

In the isolated problem (IP) approach, the potential distribution $V_{k}(1)$ is written as the sum of two parts: 1) the "isolated pontential" $U_{k}^{0}$ which is computed under the assumption that the conductivity in the $(m-1)$ th com-

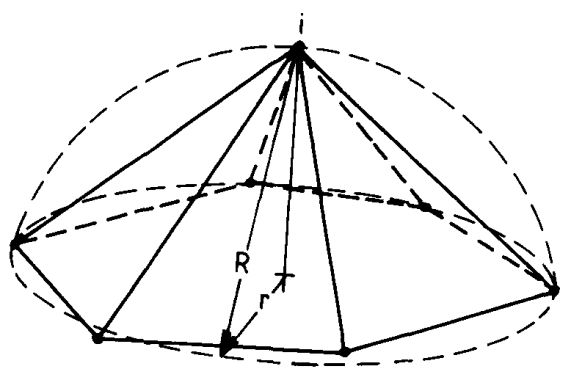

Fig. 2. The surface around a vertex point $i$ can be approximated by a spherical segment. To span this segment we use the fact that the sides opposite to vertex point $i$ of the triangles surrounding this vertex point enclose, in first order approximation, a circle with radius $r$. The distance between the vertex point $i$ and this circle is $R$.

partment is equal to zero $\left(\sigma_{m}^{+}=\sigma_{m-1}^{-}=0\right)$ and 2) the "remnant potential" $U_{k}$ being the difference between $V_{k}$ and $U_{k}^{0}$ :

$$
V_{k}\left(\boldsymbol{r}^{\prime}\right)=U_{k}^{0}\left(\boldsymbol{r}^{\prime}\right)+U_{k}\left(\boldsymbol{r}^{\prime}\right) .
$$

The isolated potential $U_{k}^{0}$ on the "outer" surfaces of the volume conductor (see Fig. 3) is zero since no sources are present and the volume currents in this outer part are also zero because $\boldsymbol{j}=\sigma \boldsymbol{E}=-\sigma \operatorname{grad} V=\boldsymbol{0}$. Consequently, the potential distribution $U_{k}^{0}$ is constant and can be chosen to be equal to zero.

$$
\begin{aligned}
U_{k}^{0}\left(\boldsymbol{r}^{\prime}\right)= & \frac{\sigma_{s}}{\bar{\sigma}_{k}^{\prime \prime}} V_{k}^{\infty}\left(\boldsymbol{r}^{\prime}\right)-\frac{1}{4 \pi \bar{\sigma}_{k}^{\prime \prime}} \sum_{l=1}^{N} \Delta \sigma_{l}^{\prime \prime} \\
& \cdot \int_{S_{l}} U_{l}^{0}(\boldsymbol{r}) d \Omega_{k l}
\end{aligned}
$$

where

$$
\begin{gathered}
U_{k}^{0}\left(\boldsymbol{r}^{\prime}\right)=0 \quad 1 \leq k<m \\
\bar{\sigma}_{k}^{\prime \prime}=\bar{\sigma}_{k} \text { and } \Delta \sigma_{k}^{\prime \prime}=\Delta \sigma_{k} \\
k \neq m \\
\sigma_{m}^{\prime \prime}=0, \text { hence } \bar{\sigma}_{m}^{\prime \prime}=\frac{1}{2} \sigma_{m}^{-} \text {and } \Delta \sigma_{m}^{\prime \prime}=\sigma_{m}^{-} \\
k=m .
\end{gathered}
$$

For a volume conductor consisting of $N$ compartments, the equation for the remnant potential $U_{k}$ can be derived and rewritten in a form analogous to 1 (see Appendix VII-B):

$$
\begin{aligned}
U_{k}\left(\boldsymbol{r}^{\prime}\right)= & \frac{\sigma_{m}^{+}}{\bar{\sigma}_{k}} U_{k}^{\infty}\left(\boldsymbol{r}^{\prime}\right)-\frac{1}{4 \pi \bar{\sigma}_{k}} \sum_{l=1}^{N} \Delta \sigma_{l} \\
& \cdot \int_{S_{l}} U_{l}\left(\boldsymbol{r}^{\prime}\right) d \Omega_{k l} \quad \text { with } \\
U_{k}^{\infty}\left(\boldsymbol{r}^{\prime}\right)= & \frac{1}{4 \pi}\left[\int_{S_{m}} U_{m}^{0}(\boldsymbol{r}) d \Omega_{k m}-2 \pi \delta_{k m} U_{m}^{0}\left(\boldsymbol{r}^{\prime}\right)\right] .
\end{aligned}
$$




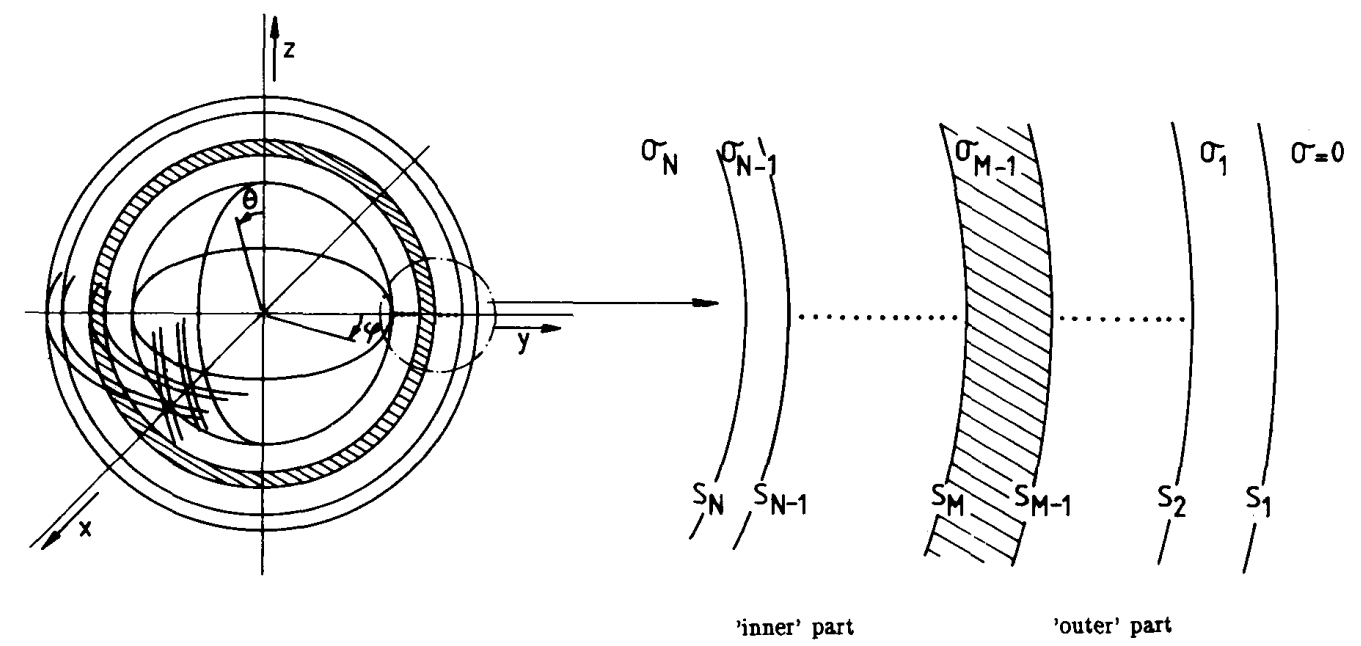

Fig. 3. Schematic representation of a volume conductor consisting of $N$, closed compartments. The compartment boundaries are, for simplicity, represented as spheres but also other arbitrary shaped ones can be used. The $(m-1)$ th compartment has a relative small conductivity and divides the whole volume conductor into an "inner" part $\left(S_{k}, k=m\right.$ up to $\left.N\right)$ and an "outer" part $\left(S_{k}, k=1\right.$ up to $\left.m-1\right)$. The Cartesian and spherical coordinate systems used are indicated in the figure.

Both (6) and (7) are Fredholm integral equations of the second kind. Consequently, the numerical methods used for solving $U_{k}^{0}$ and $U_{k}$ can be the same as those used for $V_{k}$. If more than one compartment inside the volume conductor has a relatively small conductivity, the IP approach can be used repeatedly.

By using the IP approach, the magnification of the numerical error is diminished since the volume conductor, used to compute the isolated potential $U_{k}^{0}$, does not contain any compartments with a low conductivity and the remnant potential $U_{k}$ does not show error magnification either. The last proposition can be understood by two arguments: 1) the new source term for $U_{k}$ is proportional to $\sigma_{m}^{+}(7)$ and 2) the magnitude of $U_{k}$ is small on the inner surfaces (i.e., several percent of the total potential $V_{k}$ ) and reduces when $\sigma_{m}^{+}$does, since for the computation of $U_{k}^{0}$ the conductivity $\sigma_{m}^{+}$is assumed to be zero. Thus, since both the source term and the volume term of $U_{k}$ will reduce when $\sigma_{m}^{+}$diminishes, the error magnification, which originates from the blown-up difference between the exactly known source term and the numerically computed volume term will be decreased if this IP approach is applied.

The numerical errors due to the use of the BE method are not completely eliminated. If the IP approach is incorporated in the computations they are however reduced. The potential distribution on the outer sphere of the four concentric spheres model still depends on the way in which the spherical compartment boundaries are discretized.

\section{A. Quantitative Measures}

\section{Methods}

Since the potential distributions on the outer surface of the volume conductor reflects the actual simulated and measured EEG's, the numerical errors in the simulated potential distributions on this surface will be considered. To quantify the numerical errors in these EEG's we have previously used the relative difference measure (RDM) [15] which is defined as

$$
\mathrm{RDM}=\left(\frac{\int_{S}\left(V_{R}-V\right)^{2} d S}{\int_{S} V_{R}^{2} d S}\right)^{1 / 2}
$$

where

$V_{R}$ is the reference potential distribution on the outer surface, based on the analytical (exact) expression for the single current dipole located in the four concentric spheres model,

$V$ is the potential distribution on the outer surface of the same volume conductor computed by means of the $\mathrm{BE}$ method for the same source as used for $V_{R}$, and

$S$ is the area of the map considered.

The patterns of the potential distributions will not only differ due to the use of different discretizations but the magnitudes of the numerically computed EEG's will also be blown up due to the low conductivity of the skull [5]. The differences in the shape of the potential distributions are far more important than the change in their absolute magnitude since the magnitude only reflects the strength of the source. To quantify the field differences, the RDM has been modified slightly resulting in a new overall measure, the RDM*:

$\mathrm{RDM}^{*}=\left(\int_{S}\left[\frac{V_{R}}{\sqrt{\int_{S} V_{R}^{2} d S}}-\frac{V}{\sqrt{\int_{S} V^{2} d S}}\right]^{2} d S\right)^{1 / 2}$ 
The value of this new measure is mainly determined by the differences in the patterns of the two distributions $V$ and $V_{R}$. In addition to this a so-called MAG factor, representing the overall magnification of the potential distribution $V$ will be used, defined as

$$
\mathrm{MAG}=\left(\frac{\int_{S} V^{2} d S}{\int_{S} V_{R}^{2} d S}\right)^{1 / 2}
$$

The same discrete grids are used to represent both $V$ and $V_{R}$ when the values of the RDM* and MAG [(9), (10)] are computed.

\section{B. Configuration}

The numerical errors in the boundary element method are studied by embedding a single current dipolar source in a set of four concentric spheres, which is a commonly used model of the head, and for such models analytical expressions are available to compute the electrical potential [6]. The radii of the four spheres are adapted from MRI-scans of the head of an adult, and were found to be $75,71,65$, and $63 \mathrm{~mm}$ for the spheres representing the scalp, skull, cerebro spinal fluid, and brain tissue, respectively [16]. The conductivities of the homogeneous compartments are adopted from Geddes and Baker [17] and are $0.33,0.0042,1.0$, and $0.33 \mathrm{~S} \cdot \mathrm{m}^{-1}$, respectively, the spherical compartments are homogeneously covered with 320 triangles resulting in 162 homogeneously distributed vertex points on each compartment boundary. The single current dipole, which acts as the source of the brain activity, is located at a depth of 25 $\mathrm{mm}$ if not stated else and is oriented tangential with respect to the spheres, its strength is $5 \cdot 10^{-6} \mathrm{~A} \cdot \mathrm{m}$.

\section{Discretization}

The accuracy of the numerical solution of (2) can be further improved by an overall refinement of the triangular grid, but this may result in extensive computational times [5]. To improve the numerical accuracy and nevertheless restrict the computational effort, another refining method is studied.

To study the influence of the local grid refinements on the EEG's, the same model of the volume conductor is used except for the fact that the spheres are not triangulated homogeneously. Instead, the triangular grids will be refined locally, conserving the number of discretization points used to describe the compartment boundaries. These different triangular grids are constructed by changing the location of a so-called "point of focus" $F$. Each triangular grid describing the individual compartment boundaries consists of 320 triangles and each triangle, on each compartment boundary, subtends the same solid angle with respect to the location of $\mathcal{F}$. The locations of the source and of $\mathcal{F}$ (i.e., $a$ up to $g$ ) are indicated in Fig. 4 and their coordinates are listed in Table I.

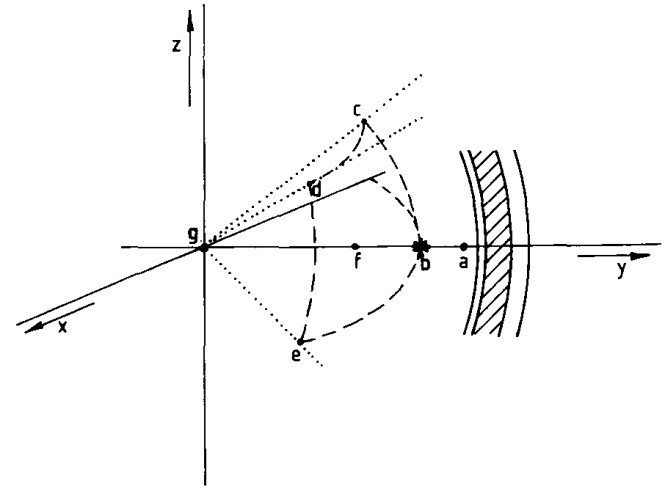

Fig. 4. The relative position of the points of focus ( $F=a$ up to $g$ ) and the source location $\left(^{*}\right)$ with respect to the four spheres model used in this paper. The locations of $\mathcal{F}$ are chosen at different positions with respect to the dipolar EEG patterns.

TABLE I

Spherical Coordinates of the Points of Focus $\mathcal{F}$ AND THE Dipolar SOURCE

\begin{tabular}{cccc}
\hline \hline & $r$ & $\theta$ & $\varphi$ \\
$\mathcal{F}$ & $(\mathrm{mm})$ & $(0)$ & $(0)$ \\
\hline$a$ & 60 & 90 & 0 \\
$b^{*}$ & 50 & 90 & 0 \\
$c$ & 50 & 45 & 0 \\
$d$ & 50 & 45 & 45 \\
$e$ & 50 & 90 & 45 \\
$f$ & 35 & 90 & 0 \\
$g$ & 0 & 0 & 0 \\
Source & 50 & 90 & 0 \\
\hline
\end{tabular}

The source location and locations of the points of focus $\mathcal{F}$ are specified by the spherical coordinates $(r, \theta$, $\varphi)$. The $(*)$ indicates that the particular point of focus coincides with the source location.

\section{The Refining Technique}

In the second, adaptive, refining technique the computation of the element $\Omega^{i j} v^{j}[(3)]$ is refined if the variation of the potential over the triangles is large. In other words, the product is recomputed if the assumption that the potential is constant on a triangle is strongly violated. The splitting criterium which determines which products $\Omega^{i j} v^{j}$ are to be recomputed is based on the source term $g$ and the matrix $B$ [see (2)]. For this adaptive recomputation method, the product $\Omega^{i j} v^{j}\left(\cong B^{i j} v^{j}\right)$ is recomputed during the iteration if criterium (11) is met:

$$
B^{i j}\left\|\nabla g^{j}\right\| \geq(1-\xi) \operatorname{MAX}\{B\|\nabla G\|\}
$$

where

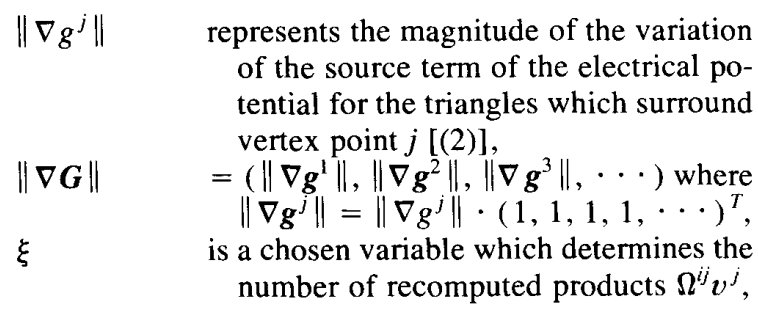




\section{$\operatorname{MAX}\{\mathfrak{K}\}$ determines the maximum element of the matrix $\mathfrak{T}$.}

Using this criterium, those products $\Omega^{i j} v^{j}$ will be recomputed for which $B^{i j}\left\|\nabla g^{j}\right\|$ has the largest values. For this adaptive recomputation method, the computation of the original product $\Omega^{i j} v^{j}$ is improved by dividing each of the $n$ triangles which surround vertex point $j$ into four smaller triangles (see Fig. 5). The potential over each smaller triangle is assumed to be the mean value of the potentials in its vertex points. The contribution of a small solid angle $\omega$ to $\Omega^{i j}$ is weighted by a factor which depends on the location of the small triangle with respect to the vertex point $j$. The improved approximation of the discrete surface integral is (see Appendix VII-C):

$$
\begin{aligned}
\int_{\otimes_{j}} V_{l}(\boldsymbol{r}) d \Omega_{k l} \approx & \frac{1}{36} \sum_{t=1}^{n}\left[4 \omega_{1}^{i t} \cdot\left(4 v^{j}+v^{j+t}+v^{j+t+1}\right)\right. \\
& +\omega_{2}^{i t} \cdot\left(v^{j}+4 v^{j+t}+v^{j+t+1}\right) \\
& +\omega_{3}^{i t} \cdot\left(v^{j}+v^{j+t}+4 v^{j+t+1}\right) \\
& +2 \omega_{4}^{i t} \cdot\left(v^{j}+v^{j+t}+v^{j+t+1}\right)
\end{aligned}
$$

where

$\otimes_{j}$ is the area attributed to vertex point $j$,

$\omega_{m}^{i t}$ is the solid angle of the $m$ th small triangle of the $t$ th split triangle surrounding vertex point $j$. For our splitting algorithm, $m$ is divided into four smaller triangles, $t=1$ through $n$ and $v^{j+n+1}=v^{j+1}$.

The refined computation of the auto-product $\Omega^{i i} v^{i}$, has to be treated different from the one of $\Omega^{i j} v^{j}$ since all triangles which surround this vertex point $i$ subtend a solid angle of $-2 \pi$, as do all the small, refined, triangles. The triangles surrounding vertex point $i$ describe, in first-order approximation, a spherical surface segment (see Fig. 2). To recompute the product $\Omega^{i i} v^{i}$, not the triangles but this dome-shaped segment will be divided into $n$ parts. The division of this segment is defined by the shape of the $n$ triangles surrounding vertex point $i$. The potential is interpolated linearly over each individual part of the segment.

By changing the variable $\xi$ in the splitting criterium (11), a percentage $P$ of the total number of products will be recomputed. For the refined computation of both the isolated and the remnant potential distributions [(6) and (7)], the same percentage $P$ of recomputed products is used.

\section{Results}

The effect of the improved $\Omega^{i i}$ approximation on the numerically computed EEG's is illustrated for the four spheres model of the head as described in Section III-B using a tangentially oriented single current dipole at a depth of $40 \mathrm{~mm}$. For this example the new, nonzero, $\Omega^{i i}$ approximation instead of the " $\Omega^{i i}=0$ " assumption results in a 10 percent reduction of the RDM value. Since this new $\Omega^{i i}$ approximation was shown to give comparable results for other discretizations of the spherical model

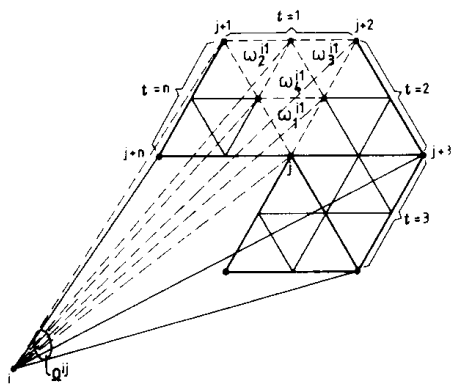

Fig. 5. When splitting criterium (11) is met for vertex point $j$, each of the $n$ surrounding vertices is divided into four smaller ones such that the sides of all of them are divided into equal parts. The original solid angle $\Omega^{i j}$, is split-up into $4 n$ smaller triangles subtending the solid angles $\omega_{m}^{\prime \prime}$ (with $m=1$ up to 4 and $t=1$ up to $m$ ). The products $\Omega^{i j} v^{j}$ are recomputed for all small triangles.

\begin{tabular}{|c|c|c|c|c|}
\hline \multirow{2}{*}{$\begin{array}{c}\text { Focus } \\
\mathfrak{F}\end{array}$} & \multicolumn{2}{|c|}{$\mathrm{BE}$ method } & \multicolumn{2}{|c|}{$\mathrm{BE} / \mathrm{IP}$} \\
\hline & $\mathrm{RDM}^{*}$ & MAG & $\mathrm{RDM}^{*}$ & MAG \\
\hline$a$ & 0.02 & 5.3 & 0.02 & 0.67 \\
\hline$b^{*}$ & 0.02 & 4.4 & 0.01 & 0.65 \\
\hline$c$ & 0.19 & 4.4 & 0.13 & 0.49 \\
\hline$d$ & 0.36 & 4.0 & 0.16 & 0.44 \\
\hline$e$ & 0.45 & 3.7 & 0.24 & 0.43 \\
\hline$f$ & 0.09 & 3.7 & 0.03 & 0.60 \\
\hline$g$ & 0.32 & 2.9 & 0.14 & 0.44 \\
\hline Mean & 0.21 & 4.1 & 0.10 & 0.53 \\
\hline SD & \pm 0.17 & \pm 0.8 & \pm 0.09 & \pm 0.11 \\
\hline
\end{tabular}

TABLE II

Values of the Measures used to Quantify the Numerical ErRors in THE COMPUTED EEG's

The RDM* and the MAG values using 1) the BE method and 2) the BE/IP method to solve the volume conduction problem for the four concentric spheres model of the head. The triangular paneling of the four spheres is determined by the location of the point of focus $\mathcal{F}$. To compute the RDM* and MAG values, the numerically computed potential distributions, based on the grid considered, are compared with the analytically computed (exact ) distributions in the same grid. The $\left({ }^{*}\right)$ indicates that the point of focus coincides with the source location. The corresponding RDM* and MAG values are printed bold. The mean values and the standard deviations (SD) are given in the last rows.

$(\mathcal{F}=a$ up to $g$ ) and much better numerical results for lower levels of discreteness, it will be used in all following simulations.

It is evident that an increase of the number of discretization points will result in a reduction of the numerical error. This effect was illustrated in an earlier study [5] in which the number of points on the four compartments of the spherical head model varied from 168 up to 320,648 , and 1280 . The numerical error was shown to be inversely proportional to the number of discretization points.

Although an acceptably small RDM value can always be obtained by an overall refinement of the grid, a local refinement of the discrete grid may also result in a reduction of the numerical errors. To present the effects of the different local grid refinements on the simulated EEG's, the numerical errors due to the use of different discrete grids are quantified by means of the RDM* and MAG values, listed in Table II(a). The potential distributions 


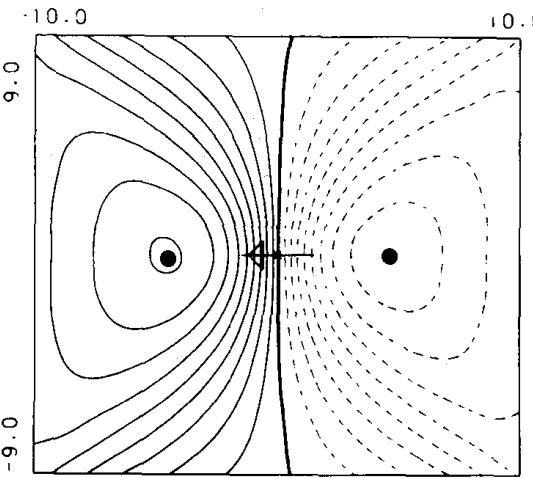

\section{analytical}

(a)

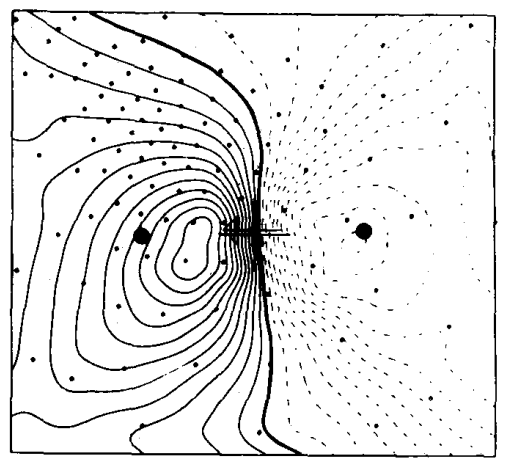

(d)

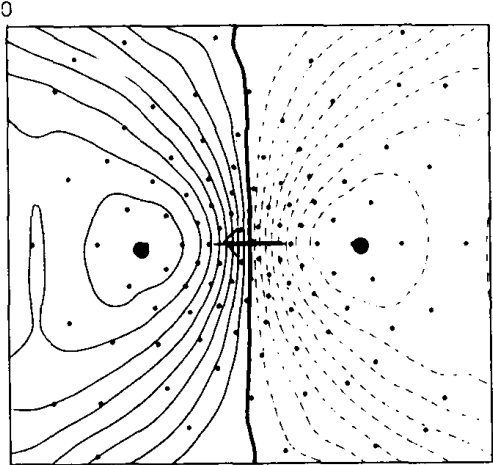

(b)

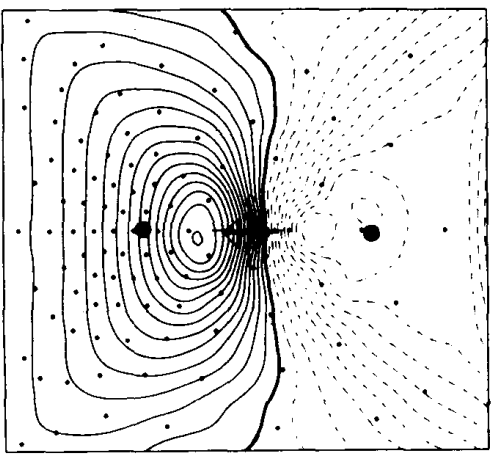

(e)

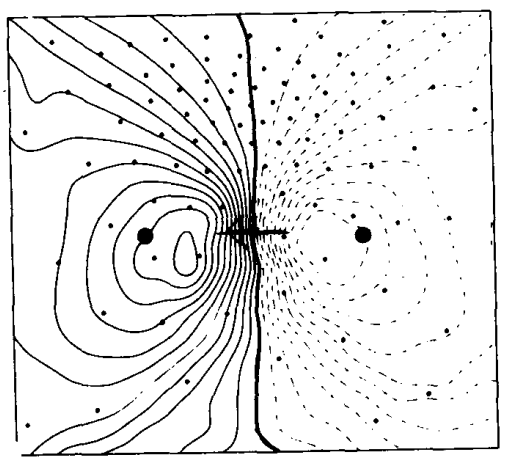

(c)

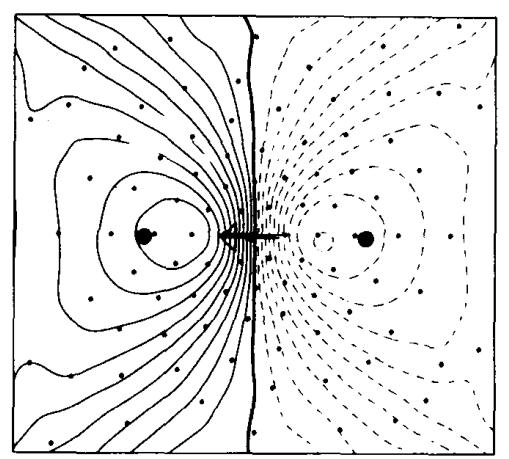

(f)

Fig. 6. The potential distributions on the outer compartment boundary of the four spheres model of the head. The first plot shows the exact potential distribution computed by means of an analytical expression and in plots (b) up to (f), the EEG's are numerically computed by means of the BE method for different triangular grids. For each plot, the same dipolar source is used as used for the first one, but now the numerical computations are based on different triangular grids describing the four spherical compartment boundaries. The small dots represent the vertices of the triangles on the outer surface and their distribution therefore identifies the different discretizations in which the successive grids are deduced from the focus locations $F$ being (b) up to (f) for Fig. 6(b) up to (f), respectively (see Fig. 4). The two large dots in each map indicate the position of the extremes in the analytic potential distributions as presented in Fig. 6(a). The cross on each map is an identical location. The arrow indicates the position and orientation of the projected source. The dotted lines indicate negative, the bold line zero and the solid lines positive potential values. The numerically computed potentials in the plots, (b) through (f), are normated which means that instead of $V, V /$ MAG is, drawn [see (10)]. The step between successive isopotential lines is $62 \mu \mathrm{V}$, the source depth is $25 \mathrm{~mm}$, and the strength of the tangentially orientated source is $5.10^{-6} \mathrm{~A} \cdot \mathrm{m}$.

on the outer surface, using a number of focus locations $\mathcal{F}$, are represented in Fig. 6 .

Since the conductivity of the skull is relatively low in comparison with that of the other compartments, the numerical errors can be reduced by applying the IP approach to the BE method. In Table II(b), the values of the quantitative measures are given for this modified BE method for the same cases as presented in Table II(a). The numerically computed EEG's using this modified BE/IP method are presented in Fig. 7. The distributions represented in this figure correspond with the same cases as presented in Fig. 6.

Table II(a) and Fig. 6 show that 1) the shape of the dipolar patterns strongly depend on the grid distribution used and are deformed more if the discretization points are distributed asymetrically with respect to the zero line of the dipolar pattern [Table II, $\mathcal{F}=d$ or $e$ and Fig. 6(d) or (e)] than if they are distributed symmetrically with re- spect to this line $(\mathfrak{F}=c)$. 2) The dipolar patterns are not deformed if the source and the point of focus coincide. 3 ) The smallest values of the RDM due to the numerical errors are an order of magnitude smaller than those which result from EEG comparisons based on different volume conductor models, which give RDM values $\mathcal{O}(0.5)$ [16]. To increase the quality of the BE method, the relative high MAG values of 4 should be decreased to a value which approximates the ideal value of one.

The numerical errors are reduced when the IP approach is incorporated in the BE method. The deformations of the numerically computed dipolar patterns, quantified by the $\mathrm{RDM}^{*}$ values in Table II, are reduced by a factor of approximately two. This can also be seen by comparing Figs. 6 and 7. Comparable results were found for other source locations and other locations of the point of focus.

The MAG factors, representing the magnification of the numerically computed potential distribution, are reduced 


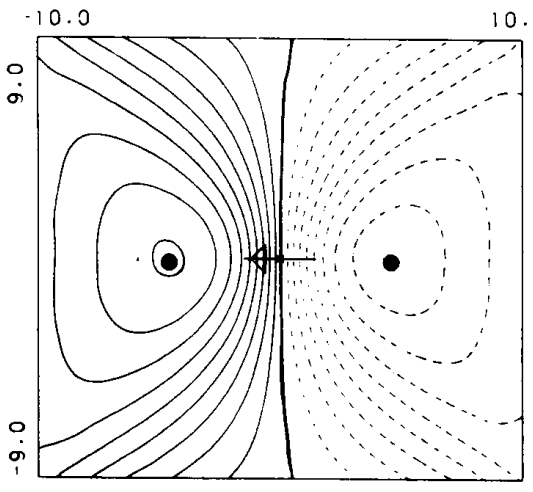

analytical

(a)

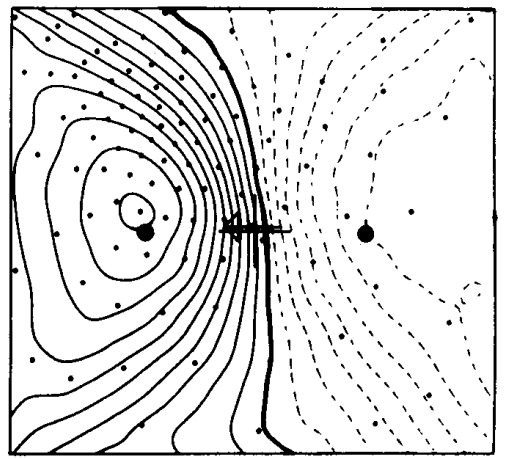

(d)

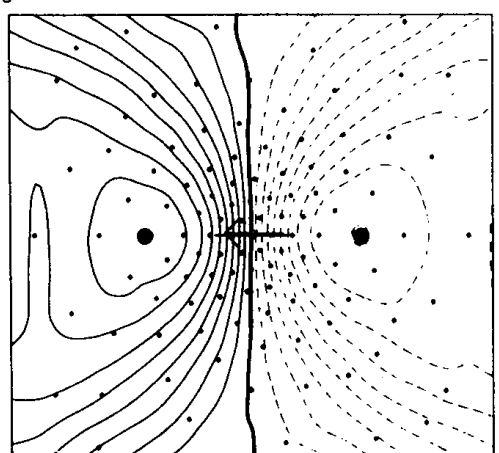

(b)

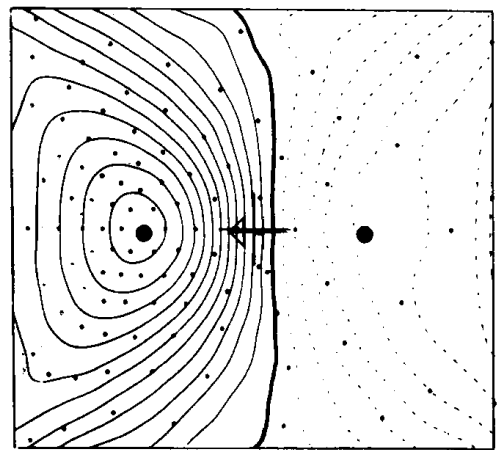

(e)

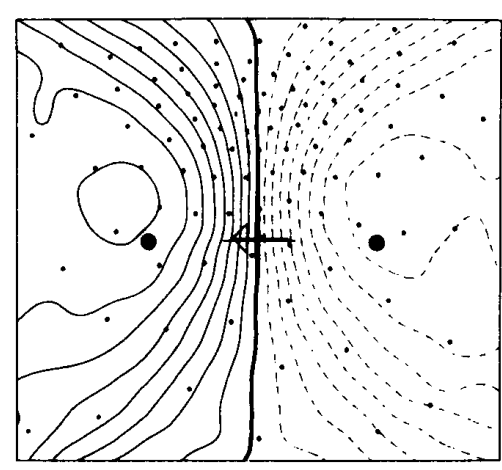

(c)

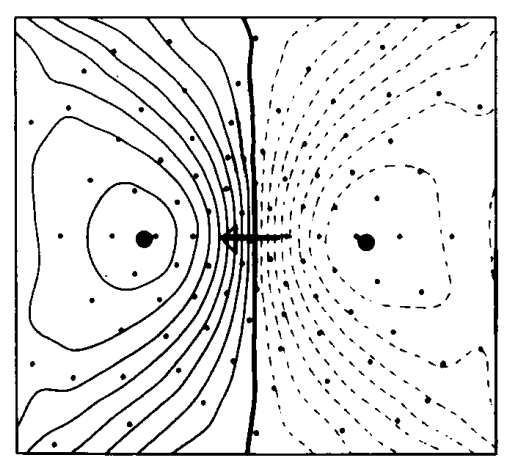

(f)

Fig. 7. As Fig. 6, but in order to compute the potential distributions, the Isolated Problem approach is incorporated in the BE method.

from a factor of 4 to a factor of 0.6 , which is, however, still too far off the ideal value of 1.0 and should be increased before this BE/IP method can be used in simulation studies involving different head models.

Finally, the effects of the refined computation of the products $\Omega^{i j} v^{j}$ on the final potential distribution are studied for the same source and the same volume conductor model as previously used. The percentage $P$ of recomputed products $\Omega^{i j} v^{j}$ is chosen to be $0,0.5,1,2,5$, and 10 percent, respectively. In Table III, the mean values of the RDM* and MAG are presented as a function of $\rho$, found by averaging the values of the measures for several locations of the point of focus $\mathcal{F}$.

Table III shows that this adaptive recomputation method will not result in further improvements of the numerical results when more than 2 percent of the products are recomputed and the improvement of the numerical accuracy is small compared with the effect of choosing different locally refined grid distributions as represented in Tables II(a) and II(b).

The potential distributions for the focus location $\mathfrak{F}=$ $d$ are visually represented in Fig. 8 for several values of the percentage $\mathcal{P}$. The effects of the recomputation of the products $\Omega^{i j} v^{j}$ for other foci and other source locations are comparable with the examples presented.
TABLE III

Mean Values of the Quantitative Measures Using Differen Percentages $\rho$ of Recomputed Products

\begin{tabular}{|c|c|c|c|c|}
\hline \multirow{2}{*}{$\frac{\mathcal{F}=}{\rho \text { (percent) }}$} & \multicolumn{2}{|c|}{$[c, d, e, g]$} & \multicolumn{2}{|c|}{$[b]$} \\
\hline & RDM $^{*}$ & MAG & $\mathrm{RDM}^{*}$ & MAG \\
\hline 0.0 & 0.17 & 0.45 & 0.01 & 0.65 \\
\hline 0.5 & 0.15 & 0.46 & 0.01 & 0.65 \\
\hline 1.0 & 0.13 & 0.47 & 0.02 & 0.65 \\
\hline 2.0 & 0.12 & 0.45 & 0.03 & 0.62 \\
\hline 5.0 & 0.14 & 0.44 & 0.08 & 0.54 \\
\hline 10.0 & 0.13 & 0.49 & 0.09 & 0.57 \\
\hline
\end{tabular}

The mean values of the RDM*s and MAG's for several values of the percentage $\beta$. To compute the means of the second and third column, the focus points $\mathcal{F}=c, d, e$, and $g$ are involved. The mean values of the RDM*s and MAG's for which the point of focus and the source location coincides are given in the last two columns ( $\mathcal{F}=b$, see Table I). Note that if the grid is chosen optimally, the recomputation method even results in an increase of the RDM* values.

\section{Discussion}

The Quantitative Measures: The main scope of this paper is to present methods which may reduce the numerical errors induced by the boundary element method. To quantify these errors, a measure is needed which should be informative and yet simple to apply. We used the RDM 


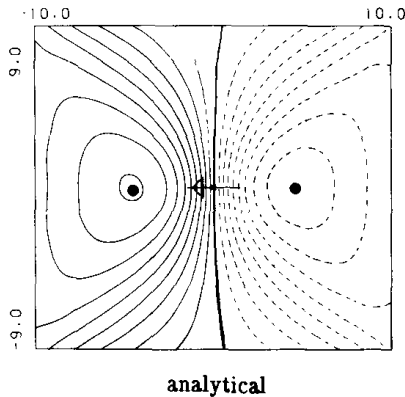

(a)

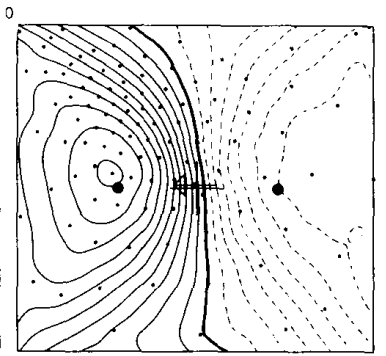

(b)

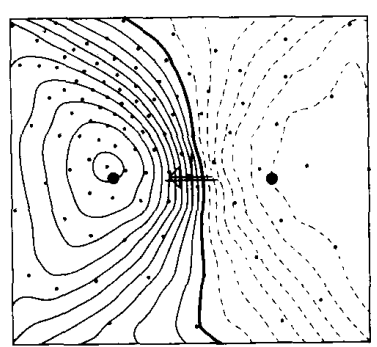

(c)

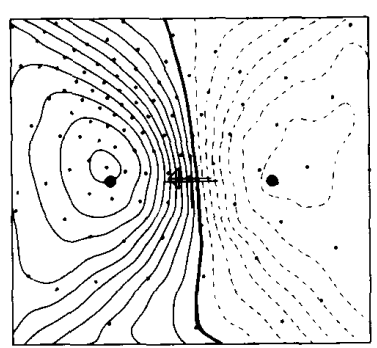

(d)

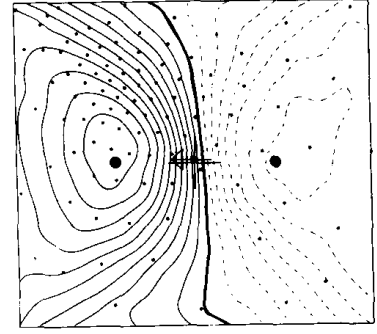

(e)

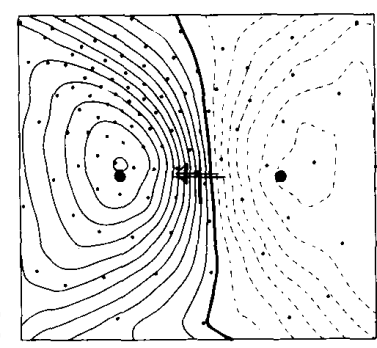

(f)

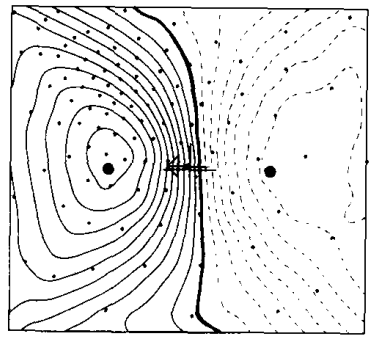

(g)

Fig. 8. Potential distributions computed by means of the BE method involving the IP approach. The point of focus is kept constant $(F=d)$ and the percentage $\rho$ of recomputed products $\Omega^{j i} v^{j}$ is varied. In the first plot, the analytically computed distribution is presented and in the following plots. the values of $\rho$ are $0,0.5,1,2,5$, and 10 percent, respectively.

in this paper since it was found to be a simple and yet very useful tool to quantify differences in the field patterns [15]. By modifying this RDM slightly, the RDM* and MAG values can be used to quantify the deformations of the magnified field patterns as well. Since the use of this measure also implies a large data reduction, some representative potential distributions are presented in the Figs. 6 up to 8 .

The Auto Solid Angle Element $\Omega^{i i}$ : Since the shape of the surface, surrounding a vertex point $i$, is approximated by a spherical surface segment, the new, nonzero approximation formula of the auto solid angle element $\Omega^{i i}$ seems exclusively suited for the study of spherical models. For other compartment models like the realistically shaped model of the head, the local approximation of a spherical segment surrounding a vertex point can also be shown to be an appropriate choice.

The Isolated Problem Approach: We prefer the isolated problem approach to reduce the pattern deformations rather than the earlier proposed Richardson extrapolation [5] since the application of this extrapolation requires that the Richardson condition is met, i.e., the patterns of the potential distributions are the same for different discretization levels [18]. Fig. 6 shows that this condition is not satisfied if the grid-distributions are not chosen optimally. However, the Richardson extrapolation technique can still be used in those cases where the location of the source is known in advance since in that case the point of focus $\mathcal{F}$ may be chosen to coincide with the source location. In this case, no deformations of the dipolar pattern occurs.
The computational time needed to solve the volume conduction problem for the four spheres model involved in this paper is proportional to $\left\{(N / 2)^{2} \log (N / 2)+N^{2}\right.$ $\log (N)\}$ if the IP approach is applied [19], whereas the numerical effort needed to reduce the numerical error by a factor of two by increasing the number of discretization points is proportional to $\left\{(2 N)^{2} \log (2 N)\right\}$. The IP approach therefore results in a reduction of the computational time by a factor of 3 for this model and this discretization level which for our VAX 8650 computer means a reduction of the CPU time from approximately 15 to 5 min for each solution of the forward problem.

In accordance with the results presented by Hämäläinen and Sarvas [8], who used spherical models which contain only one inner compartment, we have found that for such compartment models the BE method involving the IP approach results in MAG factors of approximately the ideal value of one. The residual MAG factor of 0.6 in the present paper is probably due to the fact that the surface integral of the isolated potential distribution $\left[\int_{S_{m}} U_{m}^{0}\right.$ in (7)] is reduced in magnitude. Therefore the new source term on the outer surfaces, and as a consequence the potential distribution on the outer surface, is reduced as well. We have made a separate analysis for the volume conductor consisting of only one inner compartment. Here the isolated potential distribution on the inner sphere was found to be computed with high precision, resulting in an adequate source term for $U_{k}$ and therefore a MAG factor of approximately 1 . However, the RDM* values for these volume conductors were found to be in the same order of magnitude as those presented in Table II(b). 
The Multigrid-Like Refining Technique: This technique is usually applied to numerical problems which vary strongly during the iterative process, where the panel refinements are adapted during the iterative process and based on several levels of grid refinement [20]. In our case, only the source term $g$ of the potential is used to determine which products $\Omega^{i j} v^{j}$ have to be recomputed during the iteration. This is permitted since the potential distributions do not change substantially during the iterative process. By using the proposed splitting criterium instead of a more general one, the computational effort necessary to keep track of the potential distribution during the iteration, is avoided.

An adaptive grid refining technique is essential if iterative inverse algorithms are based on the BE method since the location of the estimated source varies during such a procedure and the ultimate location of the equivalent source can only be determined appropriately if the numerical errors do not depend on the chosen grid. Another advantage of an adaptive grid refining technique, based on the splitting criterium (11), is that for realistically shaped head models those triangles, which are located close to the source will be refined automatically. In a realistically shaped head model this may be the case for the triangular panels on a fissure.

By changing the location of $\mathcal{F}$, different discrete grid distributions have been simulated while keeping the number of vertex points constant. This technique turns out to be far more effective than the adaptive recomputation method which is shown to give no appreciable reduction of the numerical errors.

The Numerical Accuracy: The accuracy of the computed electrical potentials cannot be easily determined if our realistically shaped head is involved in simulation studies because no analytical expression is available for such a volume conductor model. However, it is possible to estimate the accuracy of the numerically computed results because the numerical errors made are linearly dependent on 1) the area of the triangles and 2) the curvature of the surfaces [21]. Addendum 1): the number of points used to describe each compartment of our realistically shaped four compartment model is at least 288 which will result in a finer triangular grid than the one taken to describe the spherical models. Addendum 2): although the shape of the latter model differs from that of the tissue boundaries of our realistically shaped head model, the curvatures of the compartments of these two models are of the same order of magnitude. We may therefore assume that the numerical errors for our realistically shaped four-compartment model of the head cannot be essentially larger than the ones found in this paper and are therefore representative for those using a realistically shaped head.

\section{Conclusions}

It can be concluded from the results presented in this paper that it is desirable to eliminate the numerical errors due to the use of the BE method when this method is used to study the influence of different head models on the EEG's. Several methods are shown to be successful for improving the numerical accuracy as for instance, the handling of the auto solid angle, the isolated problem approach, and the local grid refining technique. To restrict the computational effort, an adaptive grid refining technique should be used in which a basic, coarse, grid is refined at those locations where the discretization assumptions cause the largest numerical errors.

The EEG simulations presented in this paper show that the refined computation of the products $\Omega^{i j} v^{j}$ does not noticeably improve the numerical accuracy whereas it is shown that the use of a local grid refining technique is very effective for this purpose. We therefore suggest that for future EEG simulations involving the BE/IP method an adaptive grid refining technique should be used in which extra discretization points are added to an original, coarse, grid.

\section{APpEndiXes}

\section{A. The $\Omega^{i i}$ Approximation}

The solid angle $\Omega$, subtended by the areas $A$ and $A^{\prime}$ of the spherical segments $S$ and $S^{\prime}$ at the vertex point $i$ (see Fig. 9) are by definition the same and can be computed straightforwardly:

$$
\begin{aligned}
\Omega & =\iint_{S} \frac{\boldsymbol{R}}{R^{3}} \cdot d S=-\int_{0}^{2 \pi} d \varphi \int_{\theta_{0}}^{\pi / 2} \sin (\theta) d \theta \\
& =-2 \pi \cos \left(\theta_{0}\right),
\end{aligned}
$$

with

$$
\begin{aligned}
R & =|\boldsymbol{R}|, \\
d S & =\boldsymbol{n} d S, \boldsymbol{n} \text { being the outward normal on } S, \\
\theta_{0} & =\arcsin (r / R) .
\end{aligned}
$$

When the auto solid angle element $\Omega^{i i}$ is to be approximated, we have to take into account that part of this solid angle $\Omega$ has to be attached to point $i$ and partly to the vertices which surround vertex point $i$. To include this property in the $\Omega^{i i}$ approximation formula, the area $d S^{\prime}$ is weighted by a function $f$ which is linearly dependent on the spherical coordinate $\theta$. This weighting function $f$ is equal to one for $\theta=\pi / 2$ and is zero for $\theta=\theta_{0}$. The approximation formula for the auto solid angle element is therefore determined by the surface integral A2:

$$
\begin{aligned}
\Omega^{i i} & \approx \iint_{S^{\prime}} f(\theta) \frac{\boldsymbol{R}^{\prime}}{R^{\prime 3}} \cdot d \boldsymbol{S}^{\prime} \\
& =-\int_{0}^{2 \pi} d \varphi \int_{\theta_{0}}^{\pi / 2} \frac{\left(\theta-\theta_{0}\right)}{\left(\frac{\pi}{2}-\theta_{0}\right)} \sin (\theta) d \theta \\
& =-2 \pi\left[\frac{1-\sin \left(\theta_{0}\right)}{\left(\frac{\pi}{2}-\theta_{0}\right)}\right] \\
& =-2 \pi\left[\frac{1-\cos \left(\alpha_{0}\right)}{\alpha_{0}}\right]
\end{aligned}
$$

with $\alpha_{0}=\arccos (r / R)$. 


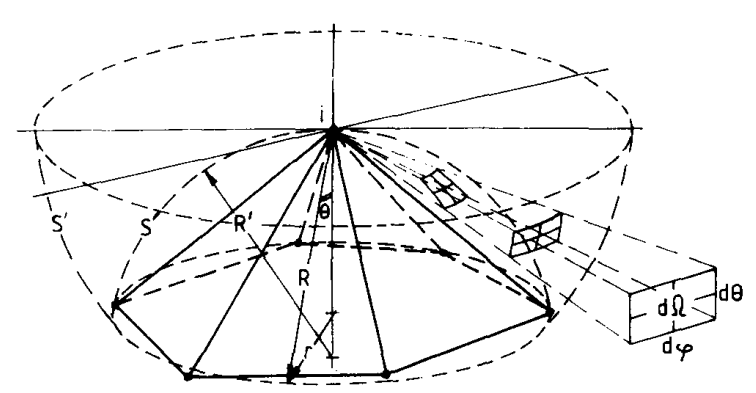

Fig. 9. The auto-solid angle element $\Omega^{i i}$ is determined by the location and shape of the triangles surrounding vertex point $i$. These triangles subtend, in first-order approximation, the same solid angle at $i$ as the spherical segment $S^{\prime}$. The infinitesimal solid angle $d \Omega$ can be assigned to $d S$ or $d S^{\prime}$ and the surface integral of (A2) can therefore be performed on $S^{\prime}$ as well.

\section{B. The Isolated Problem Approach}

The potential distribution $V_{k}$ in $\boldsymbol{r}^{\prime}$ on compartment boundary $S_{k}$ is for the IP approach written as a sum of two terms:

$$
V_{k}\left(\boldsymbol{r}^{\prime}\right)=U_{k}^{0}\left(\boldsymbol{r}^{\prime}\right)+U_{k}\left(\boldsymbol{r}^{\prime}\right)
$$

with

$U_{k}^{0}\left(r^{\prime}\right)$ the solution of the volume conduction problem involving the inner, isolated surfaces only. $U_{k}^{0}\left(\boldsymbol{r}^{\prime}\right)=0$ on the outer surfaces and $U_{k}\left(\boldsymbol{r}^{\prime}\right)$ the remnant potential on the original volume conductor.

Substitution of (A3) into (1) yields:

$$
\begin{aligned}
U_{k}\left(\boldsymbol{r}^{\prime}\right)= & \frac{\sigma_{s}}{\bar{\sigma}_{k}} V_{k}^{\infty}\left(\boldsymbol{r}^{\prime}\right)-U_{k}^{0}\left(\boldsymbol{r}^{\prime}\right)-\frac{1}{4 \pi \bar{\sigma}_{k}} \sum_{l=1}^{N} \Delta \sigma_{l} \\
& \cdot \int_{S_{l}} U_{l}(\boldsymbol{r}) d \Omega_{k l} \\
& -\frac{1}{4 \pi \bar{\sigma}_{k}} \sum_{l=m}^{N} \Delta \sigma_{l} \int_{S_{l}} U_{l}^{0}(\boldsymbol{r}) d \Omega_{k l} .
\end{aligned}
$$

Rewriting (6) results in

$$
\begin{aligned}
\frac{1}{4 \pi} & \sum_{l=m+1}^{N} \Delta \sigma_{l} \int_{S_{l}} U_{l}^{0}(\boldsymbol{r}) d \Omega_{k l} \\
& =-\bar{\sigma}_{k}^{\prime \prime} U_{k}^{0}\left(\boldsymbol{r}^{\prime}\right)+\sigma_{s} V_{k}^{\infty}\left(\boldsymbol{r}^{\prime}\right)-\frac{\sigma_{m}^{-}}{4 \pi} \int_{S_{m}} U_{m}^{0}(\boldsymbol{r}) d \Omega_{k m}
\end{aligned}
$$

where $\bar{\sigma}_{k}^{\prime \prime}=\bar{\sigma}_{k}$ for $k \neq m$ and $\bar{\sigma}_{m}^{\prime \prime}=\frac{1}{2} \sigma_{m}^{-}$.

Substitution of (A5) into (A4) yields

$$
\begin{gathered}
U_{k}\left(\boldsymbol{r}^{\prime}\right)=\frac{\sigma_{m}^{+}}{4 \pi \bar{\sigma}_{k}} \int_{S_{m}} U_{m}^{0}(\boldsymbol{r}) d \Omega_{k m}-\frac{1}{4 \pi \bar{\sigma}_{k}} \sum_{l=1}^{N} \Delta \sigma_{l} \\
\cdot \int_{S_{l}} U_{l}(\boldsymbol{r}) d \Omega_{k l}, \quad k \neq m
\end{gathered}
$$

$$
\begin{aligned}
U_{m}\left(\boldsymbol{r}^{\prime}\right)= & \frac{\sigma_{m}^{+}}{4 \pi \bar{\sigma}_{m}}\left[\int_{S_{m}} U_{m}^{0}(\boldsymbol{r}) d \Omega_{m m}-2 \pi U_{m}^{0}\left(\boldsymbol{r}^{\prime}\right)\right] \\
& -\frac{1}{4 \pi \bar{\sigma}_{m}} \sum_{l=1}^{N} \Delta \sigma_{l} \int_{S_{l}} U_{l}(\boldsymbol{r}) d \Omega_{m l} .
\end{aligned}
$$

\section{Recomputing the Surface Integrals}

When the product $\Omega^{i j} v^{j}$ is recomputed, each one of the $n$ triangles which surround vertex point $j$ is split up into four smaller triangles (see Fig. 5). To compute this product for these small triangles, the electrical potential is assumed to be a linear function over the refined triangles and the potentials on the small triangles are approximated by

$$
\begin{aligned}
& \bar{v}_{1}^{t}=\left(4 v^{j}+v^{j+t}+v^{j+t+1}\right) / 6, \\
& \bar{v}_{2}^{t}=\left(v^{j}+4 v^{j+t}+v^{j+t+1}\right) / 6, \\
& \bar{v}_{3}^{t}=\left(v^{j}+v^{j+t}+4 v^{j+t+1}\right) / 6, \\
& \bar{v}_{4}^{t}=\left(v^{j}+v^{j+t}+v^{j+t+1}\right) / 3,
\end{aligned}
$$

with

$\bar{v}_{m}^{t}$ the mean value of the potential over the $m$ th small triangle and

$t$ representing a coarse triangle having $j$ as a vertex point, $t=1$ up to $n$,

$m$ indicates a small triangle numbered from 1 up to 4 and $v^{j+n+1}=v^{j+1}$.

In the vertex approach, the solid angle $\left(\Omega^{i t}\right)$ of a triangle $t$ with respect to a point $i$, has to be attributed to the three vertex points of that triangle. To estimate the attribution of $\Omega^{i t}$ to the solid angle of vertex point $j$ of triangle $t$ (i.e., $\Omega^{i j}$ ), the area of the triangle is weighed with a linear function which is one at the vertex point $j$ and zero at the opposing vertices. A surface integral analogous to the integral in (A2) results in the approximation formula of the attribution of $\Omega^{i t}$ to the solid angle $\Omega^{i j}$.

$$
\int_{\otimes_{j}} d \Omega^{i j} \approx \frac{1}{3} \Omega^{i t} .
$$

When a triangle is split up into four smaller triangles, the attribution of the solid angles of these small triangles to the solid angle of a vertex point $j$ can be determined by using the same linear function as used to approximate $\Omega^{i j}$ in (A8). The resulting weighing factors for the solid angle contribution of the four small triangles to the vertex point $j$ are given between brackets in Fig. 10 .

The solid angle $\Omega^{i j}$ subtended by vertex point $j$ at vertex point $i$ is approximated by the sum of the weighed solid angle contributions of the $n$ sets of four small triangles:

$$
\int_{\otimes_{j}} d \Omega^{i j} \approx \frac{1}{6} \sum_{t=1}^{n} 4 \omega_{1}^{i t}+\omega_{2}^{i t}+\omega_{3}^{i t}+2 \omega_{4}^{i t}
$$

with $\omega_{m}^{i t}$ the solid angle subtended by the $m$ th small triangle of the $t$ th coarse triangle surrounding vertex point $j$ at vertex point $i$. 


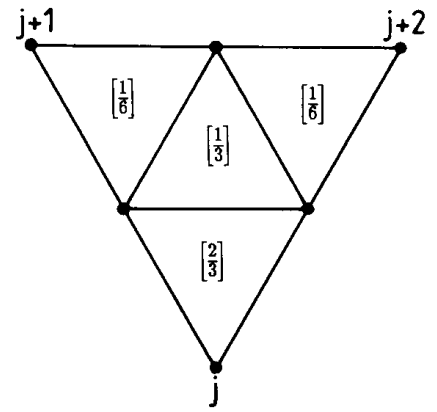

Fig. 10. The factors with which the small solid angles $\omega_{m}^{i t}(m=1 \cdots 4$ $t=1 \cdots n$, see Fig. 5) are weighted to obtain the solid angle $\Omega^{i j}$ of vertex point $j$ with respect to the vertex point $i$.

The expressions for the refined product $\Omega^{\prime \prime} v^{j}$ is found by combining (A7) and (A9):

$$
\begin{aligned}
\int_{\otimes_{j}} V_{l}(r) d \Omega_{k l} \approx & \frac{1}{6} \sum_{t=1}^{n} 4 \omega_{1}^{i t} \bar{v}_{1}^{t}+\omega_{2}^{i t} \bar{v}_{2}^{t}+\omega_{3}^{i t} \bar{v}_{3}^{t} \\
& +2 \omega_{4}^{i t} \bar{v}_{4}^{t} .
\end{aligned}
$$

\section{ACKNOWLEDGMENT}

The authors would like to thank Dr. H. B. K. Boom (Twente University) for his valuable remarks and stimulating discussions on this work.

\section{REFERENCES}

[1] C. J. Stok, "EEG/MEG single dipole source estimation," IEEE Trans. Biomed. Eng., vol. BME-34, pp. 289-296, 1987.

[2] J. W. H. Meijs, M. J. Peters, and A. van Oosterom, "Computation of MEG's and EEG's using a realistically shaped multicompartmen model of the head," Med. Biol. Eng. Comp., vol. 23 (Suppl. part 1), pp. $36-37,1985$

[3] B. He, T. Musha, S. Okamoto, Y. Nakajima, and T. Sato, "Electric dipole tracing in the brain by means of the boundary element method and its accuracy,"'IEEE Trans. Biomed. Eng., vol. BME-6, pp. 406414,1987

[4] M. S. Hämäläinen and J. Sarvas, "Feasibility of the homogeneous head model in the interpretation of neuromagnetic fields, "Phys. Med. Biol., vol. 32, pp. 91-97, 1987.

[5] J. W. H. Meijs, M.J. Peters, A. van Oosterom, and H. B. K. Boom, "The application of the Richardson extrapolation in simulation studies of EEG's," Med. Biol. Eng. Comp., vol. 25, pp. 222-226, 1987.

[6] B. N. Cuffin and D. Cohen, "Comparison of the magnetoencephalogram and the electroencephalogram," Electroencephalogr. Clin. Neurophysiol., vol. 47, pp. 132-146, 1979.

[7] J. W. H. Meijs, B. J. ten Voorde, M. J. Peters, C. J. Stok, and F. H. Lopes da Silva, "The influence of various head models on EEG's and MEG's," in Functional Brain Imaging, G. Pfurtscheller and F. H. Lopes da Silva, Eds. Toronto: H. Huber, 1988, pp. $31-$ 46.

[8] M. S. Hämäläinen and J. Sarvas, "Realistic conductivity geometry model of the human head for interpretation of neuromagnetic data," in Interpretation of Neuromagnetic Measurements: Modeling and Statistical Considerations, Ph.D. thesis, Helsinki University of Technology, Finland, 1987

[9] A. C. L. Barnard, J. M. Duck, M. S. Lynn, and W. P. Timlake, "The application of electromagnetic theory to electrocardiology II," Biophys. J., vol. 7, pp. 463-491, 1967.

[10] C. Barr, M. Ramsey III, and M. S. Spach, "Relating epicardial to body surface potential distributions by means of transfer coefficient based on geometry measurements,"' IEEE Trans. Biomed. Eng., vol. BME-24, pp. 1-11, 1977.

[11] A. van Oosterom and J. Strakee, "The solid angle of a plane triangle," IEEE Trans. Biomed. Eng., vol. BME-30, pp. 125-126, 1983

[12] M. S. Lynn and W. P. Timlake, "The use of multiple deflations in the numerical solution of singular systems of equations with appli- cations to potential theory," Siam. J. Numer. Anal., vol. 5, pp. 303 322,1968

[13] G. Dahlquist and A. Björck. Numerical Methods. Englewood Cliffs, NJ: Prentice-Hall, 1974 , p. 573.

[14] J. J. M. Cuppen and A. van Oosterom, "Model studies with the inversely calculated isochrones of ventricular depolarization," IEEE Trans. Biomed. Eng., vol. BME-31. pp. 652-659, 1984.

[15] J. W. H. Meijs, M. J. Peters, H. B. K. Boom, and F. H. Lopes da Silva, "The relative influence of model assumptions and measurement procedures in the analysis of the MEG," Med. Biol. Engng. Comp., vol. 26, pp. 136-142, 1988.

[16] J. W. H. Meijs and M. J. Peters, "The EEG and MEG, using a mode] of eccentric spheres to describe the head, ' IEEE Trans. Biomed. Eng., vol. BME-34, p. 913-920, 1987.

[17] L. A. Geddes and L. E. Baker, "The specific resistance of biological material, a compendium of data for the biomedical engineer and physiologist," Med. Biol. Eng., vol. 5, pp. 271-293, 1967.

[18] L. F. Richardson and J. A. Gaunt, "The deferred approach to the limit," Phil. Trans. R. Soc., vol. 226, pp. 299-361, 1927.

[19] H. Schippers, "Application of multigrid methods for integral equations to two problems from fluid dynamics," J. Comp. Phys, vol 48 , pp. 441-461, 1982 .

[20] A. Brandt, "Multilevel adaptive solutions to boundary-value problems," Math. Comp., vol. 31, pp. 333-390, 1977.

[21] M. S. Lynn and W. P. Timlake, "On the numerical solution of the singular integral equations of potential theory," Numer. Math., vol. 11 , pp. 77-98, 1968.

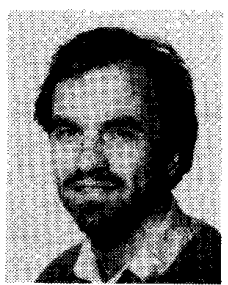

Jan W. H. Meijs was born in Heerlen. The Netherlands, on January 18,1959 . He received the M.Sc. degree in physics from the University of Eindhoven. Eindhoven. The Netherlands, in 1984. His M.Sc. thesis was on the composition of a real istically shaped multicompartment model of the head.

Since 1984 he has been with the Biomedical Engineering Division of the Twente University Enschede. The Netherlands, where he finished a $\mathrm{Ph} . \mathrm{D}$. thesis in 1988 on the influence of head geometries on MEG's and EEG's. He is currently working at the Nucletron Research Company where his main interest is on the development of radiation therapy planning systems.

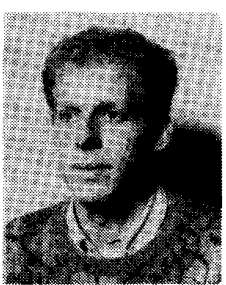

Onno W. Weier was born in the Hague. The Netherlands, on September 11, 1964. He studied electrical engineering at the University of Twente. The Netherlands, and received the M.S. degree in 1988 .

At present he is fulfilling his compulsory military service in the Audiology Group of the TNO Institute for Perception, Soesterberg. The Netherlands. His current interests include speech recognition based on neural network modeling of speech.

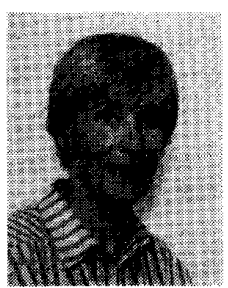

Maria J. Peters was graduated in physics from the University of Leiden. She received the Ph.D. at Twente University. Her thesis was on magnetocardiography. Her current research interests in clude the experimental and theoretical development of biomagnetism.

Adriaan van Oosterom, for a photograph and biography, see p. 391 of the March 1989 issue of this Transactions. 